\title{
SAFE AND EFFICIENT AUTONOMOUS EXPLORATION TECHNIQUE FOR 3D MAPPING OF A COMPLEX BRIDGE MAINTENANCE ENVIRONMENT
}

\author{
G. Paul, D. K. Liu, N. Kirchner and S. Webb \\ ARC Centre of Excellence for Autonomous Systems (CAS) \\ University of Technology, Sydney \\ NSW, Australia \\ \{gpaul, dkliu, nathk, swebb\}@eng.uts.edu.au
}

\begin{abstract}
This paper describes a technique for autonomously exploring a complex steel bridge environment using a 6DOF anthropomorphic robotic arm, instrumented with a laser range scanner. Potential knowledge gained from a 3D range scan at an end-effector position and orientation (pose) is estimated, then arm configurations which avoid obstacles and unknown areas are computed using an optimisation approach. Safe pose solutions are compared in terms of potential gain of new weighted-information and minimal joint movement. Both simulations and robotic platform results show exploration of unknown areas occurs in a consistent and timely manner - taking an average of $4.5 \mathrm{secs}$ to calculate the next safe valid robot arm poses. Complex environments, typical in bridge maintenance, can be explored using an anthropomorphic arm equipped with this technique.
\end{abstract}

\section{KEYWORDS}

Exploration, Collision Avoidance, Bridge Maintenance, Inverse Kinematics

\section{INTRODUCTION}

The removal of paint and rust via sandblasting, followed by the reapplication of a protective coating is an essential part of bridge maintenance: the process significantly reduces the likelihood of structurally damaging rust. However, due to newly introduced $\mathrm{OH} \& \mathrm{~S}$ regulations, it is no longer desirable for humans to perform this task. Problems arise from commonly used lead based paints and the likelihood of working in close proximity with asbestos. Thus there is little alternative other than performing a significant portion of this bridge maintenance work with a robotic system.

In order to place a robotic system in a complex environment typical of that in bridge maintenance, the system requires a method to rapidly obtain detailed on-demand surface maps [1] to facilitate robot-environment interaction. Alternate mapping solutions are not feasible: up-to-date maps are not available; manual generation of maps is prohibitively time consuming; and teleoperated based mapping/ exploration presents a high risk of collisions. As the risk of robot-environment collision is high [2], there is a need for an intelligent and efficient technique able to 'safely explore' the workspace of the robot arm and sandblasting/painting range.

Prior research into mechanised bridge maintenance where paint is removed from steel bridges by means of a teleoperated arm [3] did not address the situation where the environment is enclosed access was gained from a truck on the bridge. The focus of this work was on teleoperation rather than autonomous blasting [2] hence exploration was not required. Algorithms for obstacle detection and avoidance during path planning, as well as robot collision-free exploration techniques in $2 \mathrm{D}[4,5]$ where sensors are mounted on the mobile unit, are ineffective at solving 3D issues. Wolf et al. [6] developed a mobile unit for autonomously exploring terrain but this work focussed on localisation rather than mapping for surface interaction. 
Coverage and next best view algorithms for robot arms are active research areas but these algorithms generally do not consider potential collisions in unexplored space. Several sensor Next Best View (NBV) algorithms [7,8] were identified as possibly applicable however additional constraints on movement are also required. Additionally, NBV algorithms for object reconstruction in computer aided manufacturing/bypass-engineering generally assumed the workspace in which the sensor is moved is safe. Another example of exploration is the stair-climbing robot [9] however simplifications to $2.5 \mathrm{D}$ make it not suitable for this application. Ultimately, next view deterministic approaches do not specifically address the problem of predicting new weighted information while considering anthropomorphic arm based exploration which avoids possible collisions with both known obstacles and unknown obstacles in unexplored areas.

Inverse kinematics solutions which find suitable joint configurations are an essential part of an exploration technique for an anthropomorphic arm [10]. Importantly, different joint configurations can result in the same end-effector transform (Fig.3) hence increasing the probability of manipulator collisions - inverse kinematic approaches commonly experience local minima problems. An optimisation approach based upon $[11,12]$, if adapted by adding optimisation constraints and using various starting points - to avoid local minima - would provide a viable solution. Sujan [11] developed an innovative exploration technique but it uses stereo vision for mapping. Overall, a set of integrated solutions are required for $6 \mathrm{DOF}$ manipulator exploration without prior information including: mapping, exploration, collision avoidance and inverse kinematics.

This paper presents a technique for safe efficient 3D data collection for 3D mapping an initially unknown environment (Fig.1). The technique ensures collisions between the robotic arm and obstacles or unknown space are avoided and that the robot proactively seeks poses which will deliver maximum new data that require minimum movement to get to. Results have shown that this technique is a viable solution to our exploration problem as maps for bridge maintenance are safely acquired in a timely manner.

The breakdown of this paper is as follows: section 2 introduces the proposed exploration technique, describes how information gain is determined, how poses are selected and how movements that avoid collision are determined; and section 3 presents results from experiments conducted in a structured bridge environment.

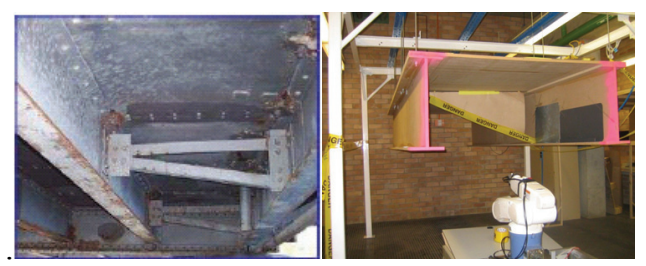

Figure 1 Left - Bridge Sandblasting Environment Right - I-beam Channel Scale Model: First Scan from Unobtrusive Position

\section{EXPLORATION TECHNIQUE}

The technique commences with the robot arm in its most unobtrusive pose; assumes completely unknown surrounds; and performs an initial scan with the fifth joint so as not to enter unexplored areas (Fig.1 right). Then, through the range, tilt and pan considerations of the laser range scanner instrument, the amount of weighted information (Fig.2) gained from a number of possible 3D scans is calculated. Different areas are multiplied by weighting coefficients (equation (1)) depending upon our desire to explore them - the workspace of the robot arm is of most importance. As can be seen in Fig.3 the end-effector can be in the same physical location with the robot in various configurations, however some configurations may cause part of the robot to enter unknown space and thus introduce the possibility for collision. The technique will then drive the robot, with assistance from a local path planner between poses [13], to a physical location that will give the system the largest amount of desired information while avoiding obstacles or unexplored space.

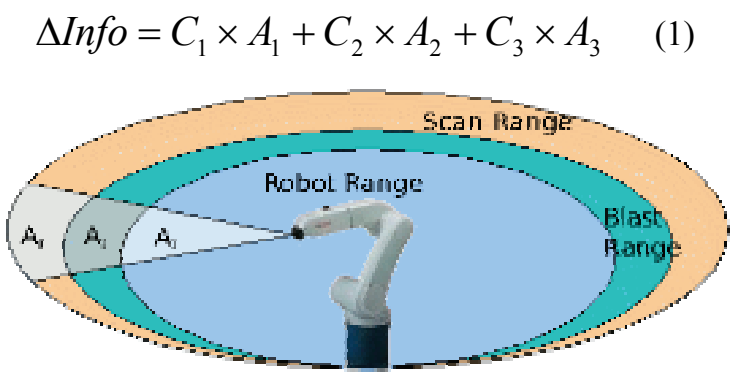

Figure 2 Areas of Importance $\left\{\begin{array}{lll}A_{1} & A_{2} & A_{3}\end{array}\right\}$ Weighted by Constants During Exploration 
The technique employs a spatial index to classify the surrounding space into known free space, known obstacles and unknown space. When range data is recognised as a surface, the data is minimised by storing bound surfaces and normals [1]. Obstacles represented by surfaces created from range data are considered, as is the pose and dimensions of the robot arm, so as to avoid issuing commands to explore from areas that endanger the robot arm.
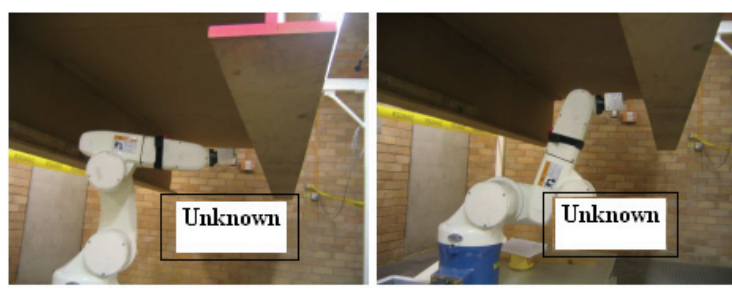

Figure 3 Joint Configurations Resulting in Same End-Effector Transform

The integrated steps of this technique (Fig.4) can be separated into: predicting information gain from different poses by considering the laser model, tilting limitations and obstacles' obscuring effect; determine joint configurations for desired endeffector poses; minimise probability of arm collisions by avoiding obstacles and unknown areas. These components are used to maximise weighted new information gained while minimising joint movement when selecting the next increment of exploration.

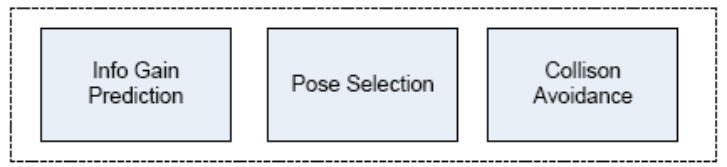

Figure 4 Processes Incorporated into Technique

\subsection{Information Gain Prediction}

Possible scan positions are chosen from a discretely sampled set of points in the robot arm's currently known workspace. In order to accurately predict $3 \mathrm{D}$ information gain a laser model was developed for the sensor constraints and capabilities: the 2D scanner field of view (max. pan angle); resolution; maximum tilt angle $\left(5^{\text {th }}\right.$ joint $)$; range; and accuracy.

The aim of this prediction step is to maximise new information (weighted by area's importance Fig.2) at each increment of exploration. As the angle of the tilt scan $\left(5^{\text {th }}\right.$ joint $)$ increases so does the: time taken; the number of points in space; and the amount of data which needs to be processed. Fig.5 shows a wireframe enclosed area to the right (blue) predicted to be found from a scan (note: in this figure the pan angle is not maximised). Where previous exploration revealed obstacles, bound plans are created using [1]. It is then possible to exclude information obscured by the obstacle to the left (red), from new information predictions. As the tilt or pan angles increase the expected area of new information increases. However, as the $5^{\text {th }}$ joint is also used to manoeuvre the 6DOF manipulator, it is desirable to constrain the movement during pose selection so as to maximise tilt angles. This is included in the objective function of the pose selection optimisation.

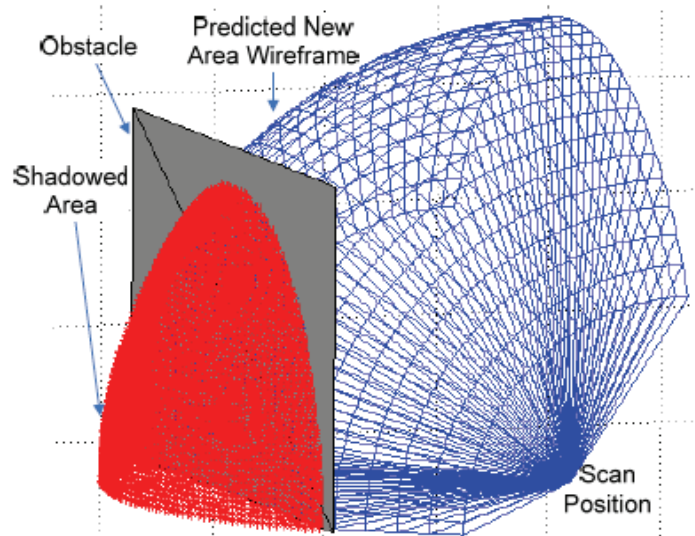

Figure 5 Visualisation of Predicted Scan Area

\subsection{Robot Pose Selection}

In the case of a simple actuator - 1 or $2 \mathrm{DOF}$, the poses for different objectives are easily determined. However in the case of a 6DOF anthropomorphic arm it is a more difficult due to local minima, joint constraints and multiple possible solutions, some with a high probability of collisions.

As the calculation of the inverse kinematic solution is relatively time consuming, rather than calculating joint configurations over a discretely sampled 3D space, we choose to calculate several possible desirable viewpoints in which to place the sensor based upon new scan information predictions, then determine poses for these. In this case the input is the transform which will position the end- 
effector (and sensor) in free space with an orientation of $\{\vec{a}, \vec{b}, \vec{c}\}$ where $\vec{c}$ represents a vector at the centre laser scan ray. Then possible joint configurations are calculated for this transform. The inverse kinematics determination requires efficiency since multiple possibilities are calculated and checked for collisions online.

An inverse kinematics optimisation approach, modified from [11,12], has been reworked to suit this application. The exploration technique passes a number of possible starting joint configuration positions (so that the inverse kinematic optimisation approach has a greater probability of avoiding local minima). Iterations and termination conditions of the optimisation are also tuned for efficiency. Termination occurs when the cost function gradient is no longer improving.

Joint limitations are also considered and poses which are outside of these limits are discarded. Equation (2) is the optimisation algorithm's error vector used to find joint configurations. It includes the distance away from the desired pose and the difference in orientation. Where $P_{d}=\left\{x_{d}, y_{d}, z_{d}\right\}$ is the desired end-effector position and $\left\{\vec{a}_{d}, \vec{b}_{d}, \vec{c}_{d}\right\}$ are the desired orientation vectors of the end-effector. The current transform of the end-effector is at position $P_{c}=\left\{x_{c}, y_{c}, z_{c}\right\}$ with orientation $\left\{\vec{a}_{c}, \vec{b}_{c}, \vec{c}_{c}\right\}$.

$$
\overrightarrow{\boldsymbol{E}}=\left[\begin{array}{c}
{[\overrightarrow{\boldsymbol{a}}, \vec{b}, \vec{c}]^{T} \cdot\left[\overrightarrow{\boldsymbol{P}}_{\boldsymbol{c}}-\overrightarrow{\boldsymbol{P}}_{\boldsymbol{d}}\right]} \\
\boldsymbol{e}^{\boldsymbol{k}_{1}\left(\sqrt{\left(\boldsymbol{t}_{\boldsymbol{d}}-\boldsymbol{t}_{\boldsymbol{c}}\right)^{2}+\left(\boldsymbol{t}_{\boldsymbol{d}}-\boldsymbol{t}_{\boldsymbol{c}}\right)^{2}+\left(\boldsymbol{t}_{\boldsymbol{d}}-\boldsymbol{t}_{\boldsymbol{c}}\right)^{2}}-\boldsymbol{C}_{\mathrm{T}}\right)} \\
\boldsymbol{e}^{\boldsymbol{k}_{2}\left(\sqrt{\left(\boldsymbol{x}_{\boldsymbol{d}}-\boldsymbol{x}_{\boldsymbol{c}}\right)^{2}+\left(\boldsymbol{y}_{\boldsymbol{d}}-\boldsymbol{y}_{\boldsymbol{c}}\right)^{2}+\left(\boldsymbol{z}_{\boldsymbol{d}}-\boldsymbol{z}_{\boldsymbol{c}}\right)^{2}}-\boldsymbol{C}_{\mathrm{A}}\right)} \\
\frac{\boldsymbol{q}_{5} \mid-\boldsymbol{C}_{\mathrm{D}}}{5}
\end{array}\right] \text { (2) }
$$

The constraint constants are as follows: $C_{T}$ is the maximum acceptable distance from the desired target; $C_{A}$ is the maximum allowable distance from the desired target and the actual target; $C_{D}$ is the maximum angle allowable off the desired orientation of the end-effector which dampens the movement of the $5^{\text {th }}$ Joint $\left(q_{5}\right)$. Since joint 5 is used to tilt the $2 \mathrm{D}$ sensor through a $3 \mathrm{D}$ scan, it is important that its range of movement is not overutilised while reaching the desired pose. In the error vector, values greater than the constants cause the error vector to become exponentially large. Overall the cost function is minimised as the actual endeffector transform gets closer to what is desired. This results in a quick (milliseconds) method to determine constrained inverse kinematic solutions.

\subsection{Collision Avoidance}

As shown in Fig.3 different joint configurations calculated from the pose selection step can result in the same end-effector transform. Therefore a collision avoidance technique closely associated with pose selection is required. Using a force field method based upon [2], all joints are encased in virtual force fields as shown in Fig.6 (only shows encasing around joints 3-6).

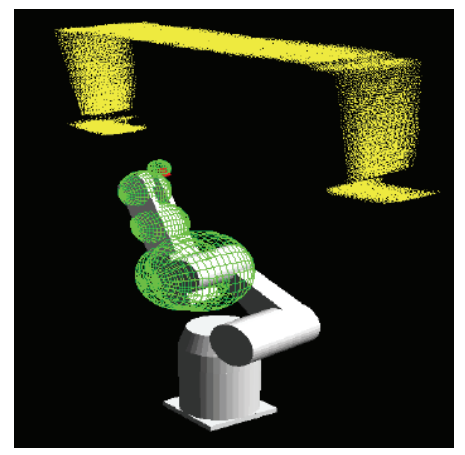

Figure 6 Virtual Force Fields Around Joints

In this case the force fields around the joints are defined at the initial configuration stage and set to JointFF for joints $\mathrm{n}=1 \rightarrow 6$. Collision avoidance checks involve performing forward kinematics on the 6DOF robot arm model using the determined joint configurations, then calculate the distance from the link $n$ center $\left\{\mathrm{x}_{\mathrm{n}}, \mathrm{y}_{\mathrm{n}}, \mathrm{z}_{\mathrm{n}}\right\}$ to the points within the robot range $\{X, Y, Z\}$ of both unknown space and obstacles.

$$
\begin{aligned}
& \forall n \in\{1 \rightarrow 6\} \\
& \text { if }\left(\sqrt{\left(x_{c}-X\right)^{2}+\left(y_{c}-Y\right)^{2}+\left(z_{c}-Z\right)^{2}} \leq J_{o \text { int } F F_{n}}\right)
\end{aligned}
$$

if the above algorithm check returns true, then a collision is possible - since one of these joint virtual force fields has unknown points/known obstacles within its defined force fields. Otherwise this is an acceptable solution. Acceptable solutions are weighted then compared so as to determine the next exploration step which has maximum predicted information gain and minimal required joint movement. 


\section{RESULTS}

Initial implementation focused on the development of a simulation (Figure 7) to check the validity of the developed technique complement experiments. This allowed for the viewing of the explored area (Figure 7 left), an essential part of collision avoidance and information gain predictions. It displays obstacles, movement of the robotic arm within the environment and information predictions.
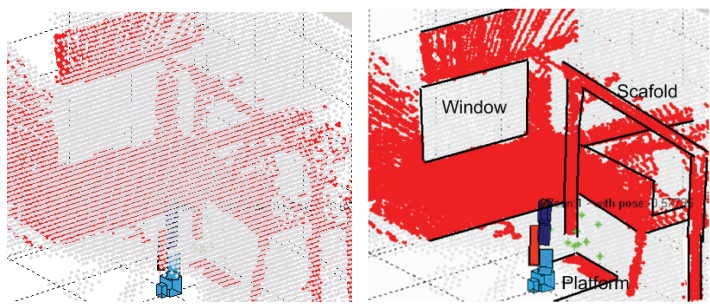

Figure 7 Environment/Robot Simulation Program

Left - Grey Points Showing Known Free Space Right - Objects Edge Highlighted, Objects Labeled

One reason for automation as opposed to teleoperation is due to human's difficult interpreting freespace (Fig.7 left in grey to highlight). Although inadequate for human participation, these points provide the system with an invaluable understanding about possible positions for the $6 \mathrm{DOF}$ manipulator. Fig.7 right highlights the edges of different objects - the window and scaffold are clear from the 3D obstacle model produced with minimal exploration.

To evaluate the technique, robotic platform experiments were setup on a scale model of the intended environment (Fig.1 right). Several experiments were designed to show the technique's ability to identify real physical space that could potentially yield the highest amount of valuable information whilst positioning the robot arm only in collision-free configurations. Fig. 8 shows the image sequence of one experiment. In this case, the robot was positioned with an obstacle (girder) in close proximity and directly above it a) and a scan was taken and environmental information gathered by the exploration technique. The position seen in b) was selected as the next best position to scan from. This result highlights the ability to consider the required physical movement of the robot as well as the potential for information gain when deciding the next position. Although intuitively, seemingly more information would be gained from scanning on the other side of the vertical partition, it is determined that the highly-weighted close proximity information remaining to be gathered on this side of the vertical partition teamed with the expense of the large robot movement makes the scan shown in b) the most efficient, yet valuable, next scan. After b) the technique moves the robot to a position on the other side of the vertical partition, shown in d), as little information remains available on this side of the partition. As can be seen in c) and d) the robot swivelled underneath the vertical partition avoiding collisions.

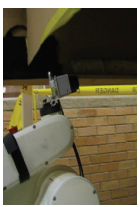

a)

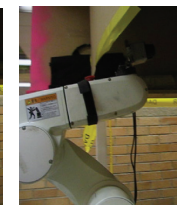

b)

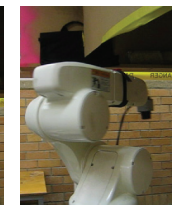

c)

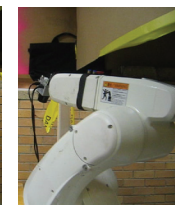

d)
Figure 8 Image Sequence of Exploration

This result clearly shows the technique's ability to select the next best view based on the amount of information available at that new position and the required movement to get to that position whilst ensuring collision avoidance.

Preliminary comparative testing found that it is extremely difficult for a remote human operator to determine possible safe configurations for the anthropomorphic robotic arm due to the difficulty in differentiating between known or unknown space. Additionally it is difficult for humans to estimate potential information gain when there are obstacles in the environment and the scanner tilt/pan angles are greater than $90^{\circ}$. Conversely preliminary results saw the technique presented here was quick (averaging $4.5 \mathrm{sec})$ and reliable $(0$ obstacle collisions) at calculating potential information from safe joint configurations, resulting in successful exploration.

\section{CONCLUSION}

The technique's ability to successfully consider and collect the data required to construct a weighted interest-area map in a time and physical movement efficient manner without the risk of collision has been experimentally verified and demonstrated. This method has been shown to be a valid solution to the critical problem of how to safely and 
efficiently collect the required data to construct an environmental map of the bridge maintenance environment. Future work will focus on continued efficiency improvement of this technique and extensive comparative testing.

\section{ACKNOWLEDGMENT}

This work is supported in part by the ARC Centre of Excellence programme, funded by the Australian Research Council (ARC) and the New South Wales State Government, and the UTS Partnership grant with the NSW Roads and Traffic Authority (RTA).

\section{REFERENCES}

[1] Paul G., Liu D.K. Kirchner N., (2007) An Algorithm for Surface Growing from Laser Scan Generated Point Clouds, Robotic Welding, Intelligence and Automation, Springer-Verlag, (in press)

[2] J. Xu, D.K. Liu, G. Fang (2006), An efficient method for collision detection and distance queries in a robotic bridge maintenance system, Robotic Welding, Intelligence and Automation, SpringerVerlag, (in press)

[3] Moon S., Bernold L.E., (1997) VisionBased Interactive Path Planning for Robotic Bridge Paint Removal, Journal of Computing in Civil Engineering 11(2): 113-120.

[4] Albers S., Kursawe K., Schuierer S., (1999) Exploring unknown environments with obstacles, Proceedings of the 10th annual ACM-SIAM symposium on Discrete algorithms. Baltimore, Maryland

[5] Yi G., Balakrishnan M., (2006) Complete coverage control for nonholonomic mobile robots in dynamic environments, Proceedings 2006 IEEE International Conference on Robotics and Automation (ICRA), pp. 1704- 1709
[6] Wolf D.F., et al. (2005) Autonomous Terrain Mapping and Classification Using Hidden Markov Models, Proceedings 2005 IEEE International Conference on Robotics and Automation (ICRA), pp. 2026- 2031

[7] Wong L.M., Dumont C., Abidi M.A., (1998) A Next Best View Algorithm for Object Reconstruction, SPIE Conf. on Sensor Fusion and Decentralized Control in Robotics Systems, Boston, MA

[8] Sanchiz J.M. Fisher R.B., (1999) A nextbest-view algorithm for $3 \mathrm{~d}$ scene recovery with 5 degrees of freedom, presented at British machine vision conference, Nottingham, UK,

[9] Okada, K., et al. (2001) Plane segment finder: algorithm, implementation and applications, in Robotics and Automation, 2001. Proceedings 2001 ICRA. IEEE International Conference Tokyo, Japan.

[10] Zhao J., Badler N.I., (1994) Inverse kinematics positioning using nonlinear programming for highly articulated figures, ACM Transactions on Graphics, vol 13 (4) (Oct) pp:313-336, ACM Press New York

[11] Sujan V.A. Dubowsky S., (2005) Efficient Information-based Visual Robotic Mapping in Unstructured Environments, The International Journal of Robotics Research, vol. 24, pp.275-293,

[12] Webb S., Furukawa T., (2006), Belief driven manipulator control for integrated searching and tracking, in IEEE/RSJ Int. Conf. on Intelligent Robots and Systems, Beijing, China, pp.4983-4988

[13] Chotiprayanakul P., Liu D.K., Wang D., Dissanayake G., (2007), A 3-Dimensional Force Field Method For Robot Collision Avoidance In Bridge Maintenance, 24th International Symposium on Automation and Robotics in Construction, India 